\title{
Early identification of recurrence in ovarian cancer: a comparison between the ovarian cancer metastasis index and CA-125 levels
}

\author{
Fei Wang ${ }^{1}$, Xuejun Zhao ${ }^{1}$, Wenhua Tan ${ }^{1}$, Wei Liu ${ }^{1}$, Yuxia Jin ${ }^{1}$, Qian Liu ${ }^{\text {Corresp. }}{ }^{1}$ \\ ${ }^{1}$ Department of gynecology and obstetrics, Second Affiliated Hospital of Harbin Medical University, Harbin, China \\ Corresponding Author: Qian Liu \\ Email address: liuqian@hrbmu.edu.cn
}

Ovarian cancer $(\mathrm{OC})$, is the second most common gynecologic malignancy. A clinical observational study was performed to investigate whether indicators that assess the risk of metastasis can identify recurrence earlier in OC patients. By successfully recruiting 41 patients with $O C$ who underwent chemotherapy, we compared cancer antigen-125 (CA125 ) and the ovarian cancer metastasis index (OCMI), which was previously developed by us in the clinic for this purpose. Our results showed that patients and their families generally took a sensible attitude toward disease progression and were willing to accept a new way to gain knowledge about the disease. Herein, the new way was the possibility of monitoring recurrence by introducing the OCMI into the clinic. Fifteen patients experienced recurrence during chemotherapy, implying treatment failure. For $53 \%$ of these patients, an abnormally high OCMI suggested a strong tendency toward metastasis at least one chemotherapy cycle prior to the pathological examination confirming recurrence. In comparison, the early recognition rate of recurrence using CA-125 levels was merely $13 \%$. Furthermore, we found that the mean values of the OCMI no longer declined after the fourth chemotherapy cycle, implying that excessive chemotherapy brings no benefit to $\mathrm{OC}$ patients. In conclusion, our findings provide a novel and feasible approach to monitor the effectiveness of chemotherapy in the treatment of $\mathrm{OC}$ by assessing the potential risk of metastasis. 
1 Early identification of recurrence in ovarian cancer: a comparison between the ovarian cancer metastasis index and CA-125 levels

3

4 Fei Wang\#, Xuejun Zhao\#, Wenhua Tan, Wei Liu, Yuxia Jin, Qian Liu*

5 Department of gynecology and obstetrics, Second Affiliated Hospital of Harbin Medical University,

6 Harbin, China.

$7 \quad$ \#The authors contributed to the work equally.

8

*To whom correspondence should be addressed. Qian Liu: Address: 246 Xuefu Road, Harbin 150086,

9 Heilongjiang Province, China; Tel/Fax: +86-451-8629-6466; E-mail: liuqian@hrbmu.edu.cn. 


\section{ABSTRACT}

23 Ovarian cancer (OC), is the second most common gynecologic malignancy. A clinical observational study was performed to investigate whether indicators that assess the risk of metastasis can identify recurrence earlier in OC patients. By successfully recruiting 41 patients with OC who underwent chemotherapy, we compared cancer antigen-125 (CA-125) and the ovarian cancer metastasis index (OCMI), which was previously developed by us in the clinic for this purpose. Our results showed that patients and their families generally took a sensible attitude toward disease progression and were willing to accept a new way to gain knowledge about the disease. Herein, the new way was the possibility of monitoring recurrence by introducing the OCMI into the clinic. Fifteen patients experienced recurrence during chemotherapy, implying treatment failure. For 53\% of these patients, an abnormally high OCMI suggested a strong tendency toward metastasis at least one chemotherapy cycle prior to the pathological examination confirming recurrence. In comparison, the early recognition rate of recurrence using CA-125 levels was merely $13 \%$. Furthermore, we found that the mean values of the OCMI no longer declined after the fourth chemotherapy cycle, implying that excessive chemotherapy brings no benefit to OC patients. In conclusion, our findings provide a novel and feasible approach to monitor the effectiveness of chemotherapy in the treatment of OC by assessing the potential risk of metastasis. 


\section{INTRODUCTION}

41 Large scale epidemiological studies have shown that ovarian cancer (OC) is the second most common

42 gynecologic malignancy, after breast cancer (Brawley, 2015; Torre et al., 2015; Siegel et al., 2017).

43 Worldwide, the number of newly diagnosed OC cases was close to 220,000 in 2008 , but this number

increased to about 240,000 in 2012 (Jemal et al., 2011; Ferlay et al., 2015). It is expected that this number

will increase further due to population aging. Despite having a lower incidence rate than breast cancer, OC

causes the largest number of gynecological cancer-associated deaths (more than 100,000 deaths per year).

One important reason is that it is difficult to detect early stage OC in a timely fashion due to the lack of

effective biomarkers for early screening (Menon et al., 2014). The relatively low incidence of OC inevitably

increases the relative cost of early screening procedures and the potential risk of erroneous identification

(Henderson et al., 2018). This raises the threshold for the screening of rational biomarkers.

Undoubtedly, the discovery of clinically available biomarkers for early screening will greatly reduce the

mortality rate of OC. Before this discovery becomes a reality, an easier and more feasible strategy is to

provide more valuable treatment management for patients who have been diagnosed with OC. Surgery plus

chemotherapy is the present mainstream OC therapeutic strategy. As about $70 \%$ of OC cases are diagnosed

at advanced stages (Jayson et al., 2014), multi-cycle chemotherapy is unavoidable. Therefore, a big

challenge is how to avoid treatment failure due to drug resistance, which is common. In the clinic, about

half of OC patients face this unfortunate outcome, undergo recurrence, and eventually succumb to the

disease (Marcus et al., 2014; Au et al., 2015). One solution is to develop prognostic biomarkers that can

detect recurrence as early as possible. A successful early warning provides doctors an invaluable 
61

62

63

64

65

66

67

recurrence have been approved by the US Food and Drug Administration: cancer antigen-125 (CA-125)

and human epididymis protein 4 (HE4). However, the effectiveness of any individual biomarker or a combination of the two has been shown to be very limited (Van Gorp et al., 2011).

According to the official definition of the International Federation of Gynecology and Obstetrics, OC can be divided into four stages, stage I to stage IV (Prat and FIGO Committee on Gynecologic Oncology, 2014). Stage I indicates no tumor cell migration event has occurred. However, the next three stages indicate an increasing range of tumor cell dissemination from the ovaries to other organs. Multi-organ metastasis often indicates a more serious disease status and lower chance of survival (Bast et al., 2009). Based on the inner logic of this definition, we developed a novel indicator, the ovarian cancer metastasis index (OCMI), which integrates CA-125 levels with six routine clinical examination indicators by a neural network cascade (NNC)-multiple linear regression hybrid model (Qu et al., 2018). NNC is an artificial neural network (ANN) with a serial data input architecture, in which multiple small ANN units that handle single input parameter are connected in tandem to complete the overall prediction task (Li et al., 2015; Hou et al., 2016; Cui et al., 2018). Our work suggested that the OCMI could successfully identify the existence and extent of multi-organ metastasis for a given patient with OC.

Metastasis suggests the existence of active cancer cells. This may further imply resistance to chemotherapeutic agents and the possibility of recurrence. Based on this reasonable hypothesis, we performed this clinical study, in which 41 patients with OC were recruited. In this study, we investigated the potential logical relationship between abnormal OCMI values and the recurrence event that is going to happen during the next period of multi-cycle chemotherapy. Such an effort was aimed to establish an association between metastasis and recurrence in OC and develop a novel biomarker for early prediction of 
82

83

84

85

86

87

88

89

90

91

recurrence.

MATERIALS AND METHODS

\section{Ethical statement}

This work was a clinical observational study that was approved by the Ethics Committee of the Second Affiliated Hospital of Harbin Medical University (Approval number: KY2017-217) and carried out in accordance with the Declaration of Helsinki. Each participant recruited in this study was informed of the project, signed a written consent form, and completed a short questionnaire (File S1). The purpose of the questionnaire survey was to identify patients' or family members' attitude about early prediction of recurrence. The family members and the patients were considered to have the same decision-making ability. In the entire study, no biological samples were collected from any patient.

\section{Inclusion and exclusion criteria}

All the patients enrolled in this study were diagnosed with epithelial ovarian cancer and underwent multicycle chemotherapy. Patients were excluded from the study if they were diagnosed with another malignant tumor or endometriosis.

\section{Patient information collection}

For each patient, the information in her medical record was use to confirm recurrence and to calculate the OCMI, following the method established previously ( $\mathrm{Qu}$ et al., 2018). The information included the patient's age, pharmacotherapeutic regimen, number of metastatic organs confirmed during surgery, ascites, laterality, imageologically-diagnosed or pathologically-confirmed recurrence, and four blood test indicators (CA-125, lymphocyte percentage, prealbumin, and blood platelet count) that were measured at 
103

104

105

106

107

108

109

110

111

112

113

114

115

116

117

118

119

120

121

122

123

the time of diagnosis, before surgery, and before each cycle of chemotherapy.

\section{Calculation of the OCMI}

OCMI values were calculated for each patient when the four blood test indicators mentioned above were available, typically at the time of diagnosis, before surgery, and before any cycle of chemotherapy. An established neural network cascade (NNC)-multiple linear regression (MLR) hybrid model (Qu et al., 2018) was directly used for this calculation. The hybrid model contained an NNC and an MLR formula. The NNC, an artificial neural network model of a serial data input architecture (Li et al., 2015; Hou et al., 2016; Cui et al., 2018), was previously built using a training dataset containing 534 patients with OC (Qu et al., 2018). Briefly, the four blood test indicators were normalized into a 0 to 1 digital number before further use, as previously described (Zhu and Kan, 2014). After that, the normalized values of the four blood test indicators were inputted into the NNC, and the NNC output was calculated using STATISTICA Neural Networks (SNN, Release 4.0E; Statsoft, Tulsa, OK, USA). Finally, the NNC output, ascites, and laterality were inputted into the MLR formula to calculate the OCMI value. If no ascites was found, the variable was valued at 0 ; otherwise, it was valued at 1 . For the laterality variable, a value of 0 represented unilateral OC and a value of 1 indicated bilateral OC.

\section{Determination and validation of metastasis discrimination thresholds}

The training dataset containing 534 patients with OC (Qu et al., 2018) was used to determine thresholds for CA-125 levels, the NNC, and the OCMI for discriminating whether metastasis occurs in a patient with OC (Table S1). MedCalc version 15.8 (MedCalc, Mariakerke, Belgium) was used, and receiver operating characteristic (ROC) curve analysis was performed to obtain the optimal thresholds of CA-125 levels, the NNC, and the OCMI. In addition to the area under the ROC curve (AUROC), the sensitivity, specificity, 
124

125

126

127

128

129

130

131

132

133

134

135

136

137

138

139

140

141

142

143

144

accuracy, and Youden index were calculated at the optimal cut-off point. The Youden index was the sum

of the sensitivity and the specificity minus 1, as defined previously (Youden, 1950). Additionally, an independent validation set of 267 patients with OC (Qu et al., 2018) was used to validate the prediction accuracies of metastasis identification using the optimal thresholds of CA-125 levels, the NNC, and the OCMI (Table S2).

\section{Statistical analyses}

All data are expressed as mean \pm standard deviation. Spearman's correlation test and Chi square test were performed using Graphpad Prism version 6.0 (GraphPad Software, Inc., La Jolla, CA, USA). MedCalc version 15.8 was applied to perform ROC curve pairwise comparison based on the methodology of DeLong et al. (DeLong et al., 1988). Differences were only considered to be significant at $p<0.001$.

\section{RESULTS}

\section{The questionnaire showed good acceptability of the OCMI from the patient aspect}

In our previous study (Qu et al., 2018), metastasis was defined that metastasis in any one of the 15 organs (bladder, diaphragm, greater omentum, internal genital organ, large intestine, liver, lymph node, mesentery, paracolic sulci, peritoneum, rectouterine fossa, small intestine, spleen, stomach, and ureter) was intraoperatively examined for a patient. And multi-organ metastasis means that more than one organs were found to have cancer metastasis in the intraoperative examination. Based on the good ability of the OCMI for identifying multi-organ metastasis in OC patients (Qu et al., 2018), we hoped to introduce the OCMI into clinical practice. This work is an exploratory study for this purpose. A questionnaire was designed and applied to investigate whether patients and patients' families can accept the OCMI-based early identification 
145

146

147

148

149

150

151

152

153

154

155

156

157

158

159

160

161

162

163

164

165

of recurrence (Supplemental File 1). A total of 22 patients and 28 patients' families were invited to

participate in the questionnaire (Figure 1A). The response rate was $82 \%$. Only 6 patients and 3 patients'

families refused to fill out the questionnaire. Finally, the necessary medical record information of 41

patients was collected to calculate the OCMI (Supplemental Table 1).

Although 16 patients and 25 patients' families agreed to participate in the study, more than two-thirds of the participants expressed doubts about the OCMI (Figure 1B). However, most of the responders expressed

the desire to receive the OCMI test results in the shortest possible time (Figure 1C). Six patients chose to face the result alone, but the majority of patients chose to consult with their families, regardless of the result

(Figure 1D). Finally, all the participants agreed to adopt the OCMI for disease management during hospitalization and after discharge.

\section{The OCMI has a stronger metastasis risk recognition ability than CA-125 levels}

A published dataset of 534 patients with OC (Qu et al., 2018) was used to set the metastasis discrimination thresholds of the OCMI and CA-125 levels (Supplemental Table 2). Our previous study had shown a greater advantage of the OCMI in identifying multi-organ metastasis than the NNC or CA-125 levels (Qu et al., 2018). When using the optimal cut-off points, the OCMI had a higher accuracy in multi-organ metastasis risk recognition than CA-125 levels (Table 1). In the present study, ROC curve comparison analysis was performed to investigate the comparative advantage of the OCMI in identifying potential metastasis risk

(Figure 2). The OCMI successfully identified metastasis better than the NNC or CA-125 levels $(p<0.001)$.

The AUROC for the OCMI was 0.856 , but that for CA-125 levels was 0.752 . When using the optimal cutoff point, the prediction accuracy of the OCMI was $77.2 \%$, which was superior to that of CA-125 levels (74.0\%; Table 2). 
166

167

168

169

170

171

172

173

174

175

176

177

178

179

180

181

182

183

184

185

186

\section{The OCMI achieves early identification of recurrence better than CA-125 levels}

For each patient, blood test indicators (CA-125, lymphocyte percentage, prealbumin, and blood platelet count) were recorded before each cycle of chemotherapy, and the OCMI was calculated using the method previously described (Qu et al., 2018). An obvious decline in the CA-125 levels was observed from the beginning of chemotherapy to the fifth chemotherapy cycle. However, after that time, the CA-125 levels no longer decreased (Figure 3A). A similar trend was observed with the OCMI (Figure 3B). During our observation period, 15 patients experienced recurrence (Supplemental Table 1). It should be noted that $60 \%$ of the recurrence events happened at the seventh chemotherapy cycle, whereas no recurrence was observed from the beginning of chemotherapy to the fifth chemotherapy cycle. By using multi-organ metastasis discrimination thresholds $(\mathrm{CA}-125=376 \mathrm{U} / \mathrm{mL}$ and $\mathrm{OCMI}=0.558), 2$ patients had early identification of recurrence by CA-125 levels, but 6 had early identification using the OCMI. Following the metastasis discrimination thresholds $(\mathrm{CA}-125=313 \mathrm{U} / \mathrm{mL}$ and $\mathrm{OCMI}=0.504), 2$ patients who underwent recurrence were identified early by CA-125 levels, but 8 were identified early by the OCMI (Figure 3C). On the contrary, among the 26 non-recrudescent patients, only 3 had OCMI values higher than 0.504 from the beginning of chemotherapy to the fifth chemotherapy cycle. Comparatively, 4 non-recrudescent patients had CA-125 levels higher than $313 \mathrm{U} / \mathrm{mL}$ during the same period.

\section{DISCUSSION}

Due to the lack of effective early monitoring tools for OC recurrence, we can only take remedial measures after patients develop recurrence (Davidson and Tropé, 2014; Au et al., 2015; Nezhat et al., 2015; Bowtell et al., 2015). In this exploratory work, we attempted to establish a link between metastasis and recurrence 
using a quantitative index that was developed to assess the degree of metastasis in OC patients (Qu et al., 2018). The OCMI is an integrated index generated from six conventional clinical examination indicators.

One advantage of not developing a separate and new clinical test is that it can be immediately brought into clinical practice. According to the definition of the index, a greater OCMI indicates a wider range of metastasis or a higher metastasis risk. In the present study, we further confirmed that the OCMI could identify recurrence early by identifying patients at high risk of metastasis. It has been generally accepted that metastasis is the main indicator of the pathological development of OC (Bast et al., 2009; Prat and FIGO Committee on Gynecologic Oncology, 2014). This is also the logical basis for this study. Several limitations of this study should be taken into consideration. One limitation is that the sample size of this single center study is small, and therefore, the conclusions made here need to be re-validated in a larger multicenter study in the future. Another limitation is that the early recognition rate of recurrence by the OCMI is still not satisfactory, and further improvement of the OCMI is needed in the future. One feasible direction is to integrate more useful indicators into the calculation of the OCMI.

Suffering from a malignant tumor causes patients to experience a greater depth of loneliness, but does not generally cause them to be overly pessimistic (Çıracı et al., 2016; Thieme et al., 2017). Consistently, our findings suggest that the vast majority of OC patients and their families could face the disease rationally when a new possibility appeared. They chose to accept it tentatively instead of rejecting it. Active participation of the vast majority of OC patients and their families implies an embodiment of optimism rather than passive acceptance due to excessive pessimism. Although our method was not able to provide a completely new and promising treatment, patients still wanted to know their OCMI test results as early as possible. This tendency indicates that good treatment and deep understanding of the disease are equally 
208

209

210

211

212

213

214

215

216

217

218

219

220

221

222

223

224

225

226

227

228

important to OC patients. Undoubtedly, valuable information can help them make decisions that are more in line with their interests. Throughout the study, we were always aware of patients' desire for valuable information about their disease condition.

On the one hand, the lack of necessary disease assessment information often leads doctors to tentatively implement overtreatment; on the other hand, patients' psychological acceptance is often underestimated by doctors and therefore it is difficult for patients to get information that is necessary for disease selfassessment. This constitutes a vicious cycle. Overtreatment will undoubtedly maximize patients' harm and minimize the benefit of clinical treatment for cancer (Loeb et al., 2014; Mukhtar et al., 2015). When a malignant disease, such as $\mathrm{OC}$, has a high probability of treatment failure, disease assessment information is more meaningful for patients. This helps them have more opportunities to rationally design their remaining time and make it more valuable.

In our study, we considered two threshold standards for the OCMI. A high threshold (OCMI $=0.558)$ was produced from a previous ROC curve analysis for identifying multi-organ metastasis. Our result indicates that only $40 \%$ of the patients with recurrence could be identified in advance of five chemotherapy cycles. By using the MedCalc software, a lower threshold $(\mathrm{OCMI}=0.504)$ was generated in this study from a ROC curve analysis for identifying metastasis, rather than multi-organ metastasis. Our finding indicates that application of the lower threshold of OCMI led to a higher recognition rate of patients with recurrence. Eight of the 15 patients with recurrence were identified in advance of at least one chemotherapy cycle. Meanwhile, only 3 of the 26 patients without recurrence were suggested to have a high risk of relapse. Comparatively, use of CA-125 alone, a specific biomarker of OC, failed to identify patients with recurrence before the recurrence was confirmed by clinicopathological examination. The early recognition rate of 
229

230

231

232

recurrence was 13\% using CA-125 levels as a biomarker. Furthermore, our findings also suggest that increasing the number of chemotherapy cycles did not further reduce the risk of cancer metastasis in OC patients. Clinical observations demonstrated that patients could only show a limited response to overchemotherapy (Bowtell et al., 2015) and over-chemotherapy led to a greater possibility of recurrence (Agarwal and Kaye, 2003). Our findings further suggest that over-chemotherapy does not provide more benefits in blocking cancer metastasis than moderate chemotherapy does.

In conclusion, we provide a new and useful tool for the early identification of relapse during chemotherapy administration in OC patients. Our findings suggest that model refining of only a few of routine clinical indicators can bring a new possibility of early prediction of cancer recurrence. More importantly, this method means a valuable opportunity to replace the failed chemotherapy regimen before recurrence really occurs. We believe that introducing the OCMI into clinical practice represents a feasible and low-cost strategy and the OCMI can fill in the information shortage of clinical disease management of OC, which is caused by low efficiency of CA- 125.

\section{REFERENCES}

Agarwal R, Kaye SB. 2003. Ovarian cancer: strategies for overcoming resistance to chemotherapy. Nature Reviews Cancer 3(7):502-516 DOI 10.1038/nrc1123.

Au KK, Josahkian JA, Francis JA, Squire JA, Koti M. 2015. Current state of biomarkers in ovarian cancer prognosis. Future Oncology 11(23):3187-3195 DOI 10.2217/fon.15.251.

Bast RC Jr, Hennessy B, Mills GB. 2009. The biology of ovarian cancer: new opportunities for translation. Nature Reviews Cancer 9(6):415-428 DOI 10.1038/nrc2644. 
250

251

252

253

254

255

256

257

258

259

260

261

262

263

264

265

266

267

268

269

270

Bowtell DD, Böhm S, Ahmed AA, Aspuria PJ, Bast RC Jr, Beral V, Berek JS, Birrer MJ, Blagden S,

Bookman MA, Brenton JD, Chiappinelli KB, Martins FC, Coukos G, Drapkin R, Edmondson R,

Fotopoulou C, Gabra H, Galon J, Gourley C, Heong V, Huntsman DG, Iwanicki M, Karlan BY, Kaye

A, Lengyel E, Levine DA, Lu KH, McNeish IA, Menon U, Narod SA, Nelson BH, Nephew KP,

Pharoah P, Powell DJ Jr, Ramos P, Romero IL, Scott CL, Sood AK, Stronach EA, Balkwill FR. 2015.

Rethinking ovarian cancer II: reducing mortality from high-grade serous ovarian cancer. Nature Reviews

Cancer 15(11):668-679 DOI 10.1038/nrc4019.

Brawley O W. 2015. Ovarian cancer prevention: Time for primetime? Cancer 121(13):2121-2123 DOI

10.1002/cncr.29347.

Çıracı Y, Nural N, Saltürk Z. 2016. Loneliness of oncology patients at the end of life. Support Care Cancer 24(8):3525-3531 DOI 10.1007/s00520-016-3159-5.

Cui X, Li Z, Zhao Y, Song A, Shi Y, Hai X, Zhu W. 2018. Breast cancer identification via modeling of peripherally circulating miRNAs. PeerJ 6:e4551 DOI 10.7717/peerj.4551.

Davidson B, Tropé CG. 2014. Ovarian cancer: diagnostic, biological and prognostic aspects. Womens Health (Lond) 10(5):519-533 DOI 10.2217/whe.14.37.

DeLong ER, DeLong DM, Clarke-Pearson DL. 1988. Comparing the areas under two or more correlated receiver operating characteristic curves: a nonparametric approach. Biometrics 44(3):837-845 DOI $10.2307 / 2531595$.

Ferlay J, Soerjomataram I, Dikshit R, Eser S, Mathers C, Rebelo M, Parkin D M, Forman D, Bray F. 2015. Cancer incidence and mortality worldwide: sources, methods and major patterns in GLOBOCAN 2012. International Journal of Cancer 136(5):E359-E386 DOI 10.1002/ijc.29210. 
271

272

273

274

275

276

277

278

279

280

281

282

283

284

285

286

287

288

289

290

291

Henderson JT, Webber EM, Sawaya GF. 2018. Screening for Ovarian Cancer: Updated Evidence Report and Systematic Review for the US Preventive Services Task Force. JAMA 319(6):595-606 DOI 10.1001/jama.2017.21421.

Hou S, Wang J, Li Z, Wang Y, Wang Y, Yang S, Xu J, Zhu W. 2016. Five-descriptor model to predict the chromatographic sequence of natural compounds. Journal of Separation Science 39(5):864-872 DOI $10.1002 /$ jssc. 201501016.

Jayson GC, Kohn EC, Kitchener HC, Ledermann JA. 2014. Ovarian cancer. Lancet 384(9951):13761388 DOI 10.1016/S0140-6736(13)62146-7.

Jemal A, Bray F, Center MM, Ferlay J, Ward E, Forman D. 2011. Global cancer statistics. $C A: A$ Cancer Journal for Clinicians 61(2):69-90 DOI 10.3322/caac.20107.

Li Z, Li Y, Sun L, Tang Y, Liu L, Zhu W. 2015. Artificial neural network cascade identifies multi-P450 inhibitors in natural compounds. PeerJ 3:e1524 DOI 10.7717/peerj.1524.

Loeb S, Bjurlin MA, Nicholson J, Tammela TL, Penson DF, Carter HB, Carroll P, Etzioni R. 2014. Overdiagnosis and overtreatment of prostate cancer. European Urology 65(6):1046-1055 DOI 10.1016/j.eururo.2013.12.062.

Marcus CS, Maxwell GL, Darcy KM, Hamilton CA, McGuire WP. 2014. Current approaches and challenges in managing and monitoring treatment response in ovarian cancer. Journal of Cancer 5(1):2530 DOI 10.7150/jca.7810.

Menon U, Griffin M, Gentry-Maharaj A. 2014. Ovarian cancer screening-current status, future directions. Gynecologic Oncology 132(2):490-495 DOI 10.1016/j.ygyno.2013.11.030.

Nezhat FR, Apostol R, Nezhat C, Pejovic T. 2015. New insights in the pathophysiology of ovarian cancer 
292 293 294 295 296 297 298 299 300 301 302 303 304 305 306 307 308 309

and implications for screening and prevention. American Journal of Obstetrics \& Gynecology 213(3):262-

267 DOI 10.1016/j.ajog.2015.03.044.

Mukhtar RA, Wong JM, Esserman LJ. 2015. Preventing Overdiagnosis and Overtreatment: Just the Next Step in the Evolution of Breast Cancer Care. Journal of the National Comprehensive Cancer Network 13(6):737-743 DOI 10.6004/jncen.2015.0088.

Prat J, FIGO Committee on Gynecologic Oncology. 2014. Staging classification for cancer of the ovary, fallopian tube, and peritoneum. International Journal of Gynecology \& Obstetrics 124(1):1-5 DOI 10.1016/j.ijgo.2013.10.001.

Qu Y, He Y, Li Z, Chen X, Liu Q, Zou S, Kong C, Liu Y, Gao C, Zhang G, Zhu W. 2018. Constructing an ovarian cancer metastasis index by dissecting medical records. Oncotarget 8(60):102212-102222 DOI 10.18632/oncotarget.22336.

Siegel RL, Miller KD, Jemal A. 2017. Cancer Statistics, 2017. CA: A Cancer Journal for Clinicians 67(1):7-30 DOI 10.3322/caac.21387.

Thieme M, Einenkel J, Zenger M, Hinz A. 2017. Optimism, pessimism and self-efficacy in female cancer patients. Japanese Journal of Clinical Oncology 47(9):849-855 DOI 10.1093/jjco/hyx079.

Torre LA, Bray F, Siegel RL, Ferlay J, Lortet-Tieulent J, Jemal A. 2015. Global cancer statistics, 2012. CA: A Cancer Journal for Clinicians 65(2):87-108 DOI 10.3322/caac.21262.

Van Gorp T, Cadron I, Despierre E, Daemen A, Leunen K, Amant F, Timmerman D, De Moor B, Vergote I. 2011. HE4 and CA125 as a diagnostic test in ovarian cancer: prospective validation of the Risk of Ovarian Malignancy Algorithm. British Journal of Cancer 104(5):863-870 DOI 10.1038/sj.bjc.6606092. Youden WJ. 1950. Index for rating diagnostic tests. Cancer 3(1):32-35 DOI 10.1002/1097- 
313

314

315

316

\section{FIGURE LEGENDS}

318 antigen-125. metastasis.

0142(1950)3:1<32::AID-CNCR2820030106>3.0.CO;2-3.

Zhu W, Kan X. 2014. Neural network cascade optimizes microRNA biomarker selection for nasopharyngeal cancer prognosis. PLoS One 9(10):e110537 DOI 10.1371/journal.pone.0110537.

Figure 1. Results of the questionnaire. A. Distribution of questionnaire respondents. B. Respondents' attitudes toward the OCMI. C. The time-cost of the OCMI result. D. Persons that were informed of the OCMI result. OCMI: ovarian cancer metastasis index.

Figure 2. AUROC comparison results. NNC: neural network cascade; OCMI: ovarian cancer metastasis index, AUROC: area under the receiver operating characteristic curve.

Figure 3. Comparison between CA-125 levels and the $\mathrm{OCMI}$ in the early identification of recurrence. Variation tendencies of CA-125 levels (A) and the OCMI (B). C. Scatter plots of CA-125 values and OCMI values in recurrent patients (five chemotherapy cycles before recurrence). The left dotted line represents the CA-125 metastasis threshold $(\mathrm{CA}-125=313 \mathrm{U} / \mathrm{mL})$ and the right dotted line indicates the metastasis threshold using the OCMI $(\mathrm{OCMI}=0.504)$. OCMI: ovarian cancer metastasis index, CA-125: cancer

Table 1. Receiver operating characteristic curve analysis results for identifying multi-organ

Table 2. Receiver operating characteristic curve analysis results for identifying metastasis. 


\section{Supplementary data}

335 Supplementary File 1. Questionnaire on using the OCMI to identify the recurrence risk in ovarian

336 cancer.

337 Supplementary Table 1. Information of patients included in the study.

338 Supplementary Table 2. The dataset of ovarian cancer metastasis. 


\section{Table $\mathbf{1}$ (on next page)}

Receiver operating characteristic curve analysis results for identifying multi-organ metastasis. 
1 Table 1. Receiver operating characteristic curve analysis results of identifying multi-organ

2 metastasis.

\begin{tabular}{|l|l|l|l|l|l|}
\hline Index & Threshold & Sensitivity (\%) & Specificity (\%) & Youden index J & Accuracy (\%) \\
\hline CA-125 & 376 & 80.6 & 52.3 & 0.329 & 66.5 \\
\hline NNC & 0.565 & 71.3 & 69.9 & 0.412 & 70.6 \\
\hline OCMI & 0.558 & 84.0 & 68.8 & 0.528 & 76.4 \\
\hline
\end{tabular}

3 


\section{Table 2 (on next page)}

Receiver operating characteristic curve analysis results for identifying metastasis. 
1 Table 2. Receiver operating characteristic curve analysis results of identifying metastasis.

\begin{tabular}{|l|l|l|l|l|l|}
\hline Index & Threshold & Sensitivity (\%) & Specificity (\%) & Youden index & Accuracy (\%) \\
\hline CA-125 & 313 & 75.8 & 65.6 & 0.414 & 74.0 \\
\hline NNC & 0.458 & 78.3 & 65.6 & 0.439 & 75.8 \\
\hline OCMI & 0.504 & 75.6 & 85.4 & 0.607 & 77.2 \\
\hline
\end{tabular}

2 


\section{Figure 1}

Results of the questionnaire.

A. Distribution of questionnaire respondents. B. Respondents' attitudes toward the OCMI. C. The time-cost of the OCMI result. D. Persons that were informed of the OCMI result. OCMI: ovarian cancer metastasis index. 
A

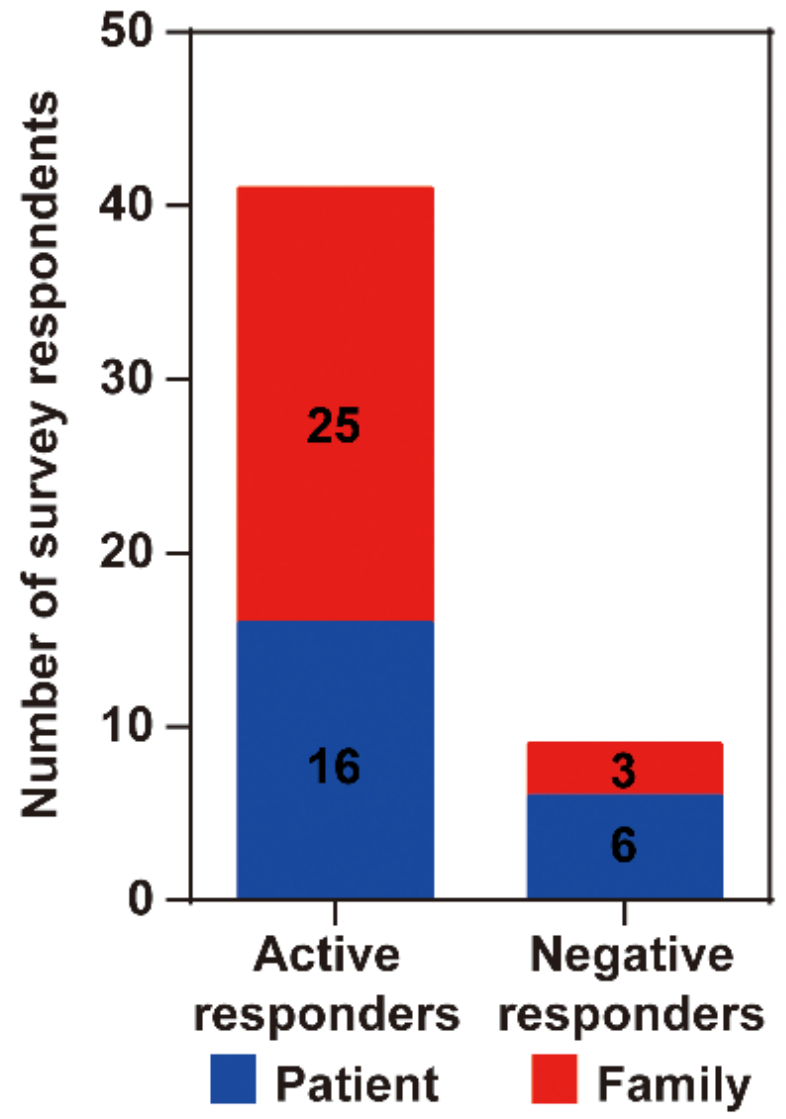

C

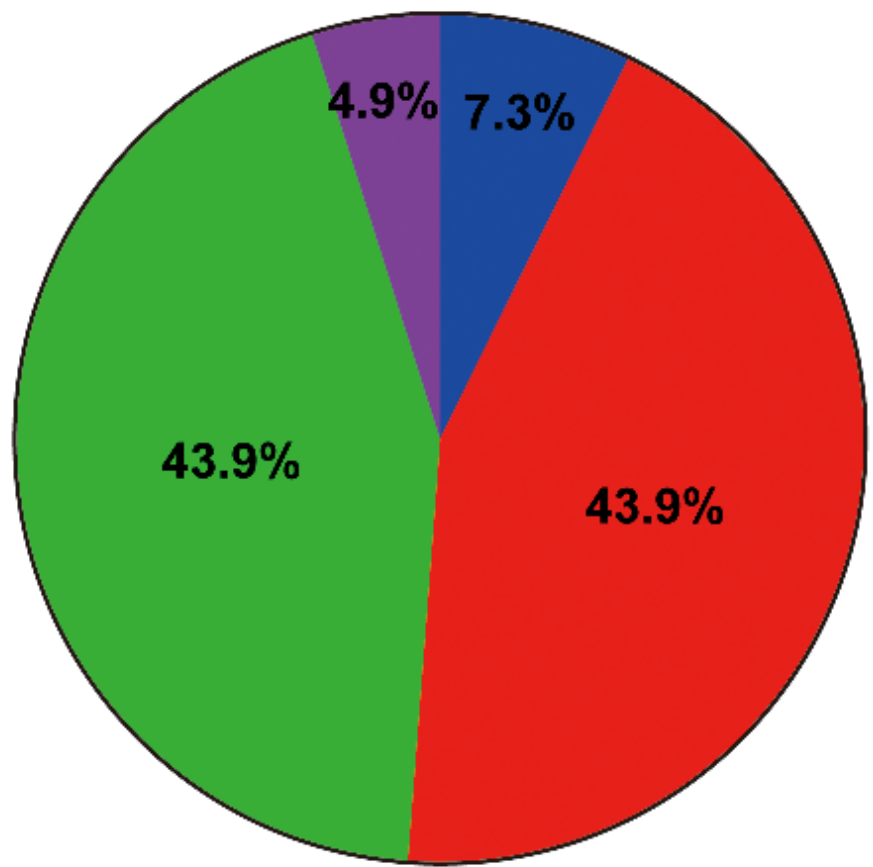

One day

Two or three days

One week One month
B

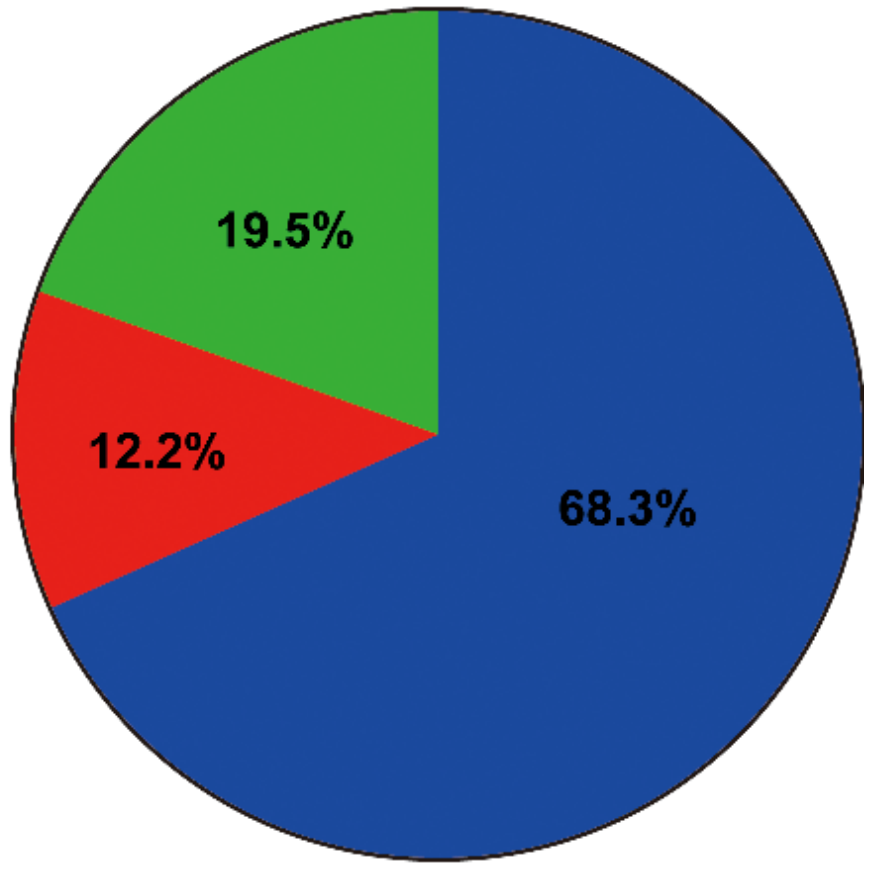

Being a rational decision-maker Taking OCMI as a valuable tool

Being accompanied by worries

D

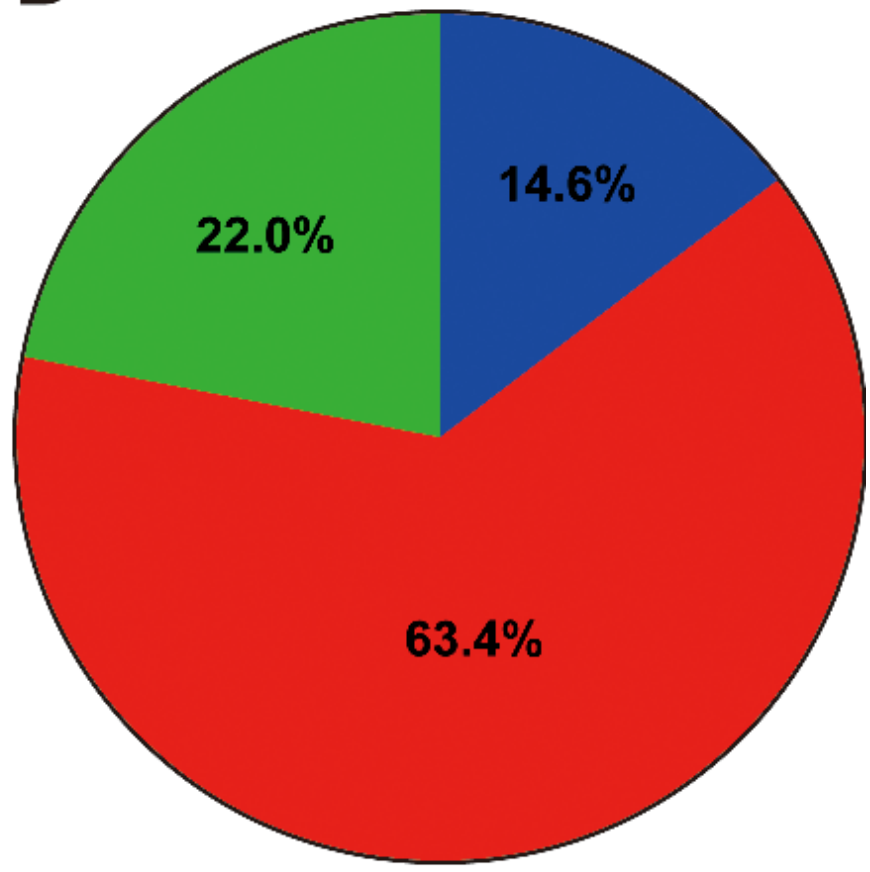

Only the patient herself

Only the families

Patient and the families 
Figure 2

AUROC comparison results.

NNC: neural network cascade; OCMI: ovarian cancer metastasis index, AUROC: area under the receiver operating characteristic curve.

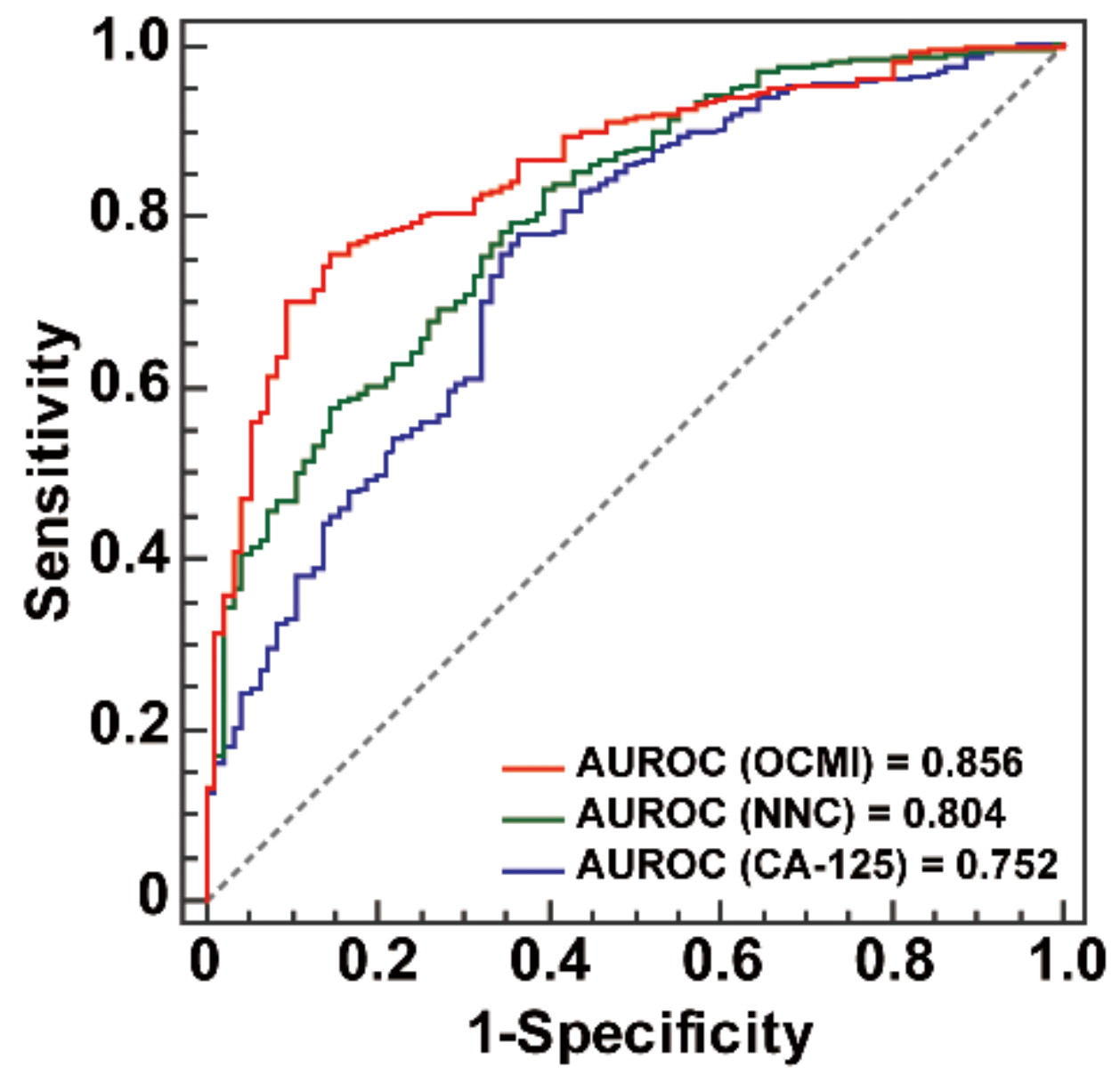




\section{Figure 3}

Comparison between CA-125 levels and the OCMI in the early identification of recurrence.

Variation tendencies of CA-125 levels (A) and the OCMI (B). C. Scatter plots of CA-125 values and OCMI values in recurrent patients (five chemotherapy cycles before recurrence). The left dotted line represents the CA-125 metastasis threshold (CA-125 $=313 \mathrm{U} / \mathrm{mL}$ ) and the right dotted line indicates the metastasis threshold using the OCMI (OCMI $=0.504)$. OCMI: ovarian cancer metastasis index, CA-125: cancer antigen-125. 
A

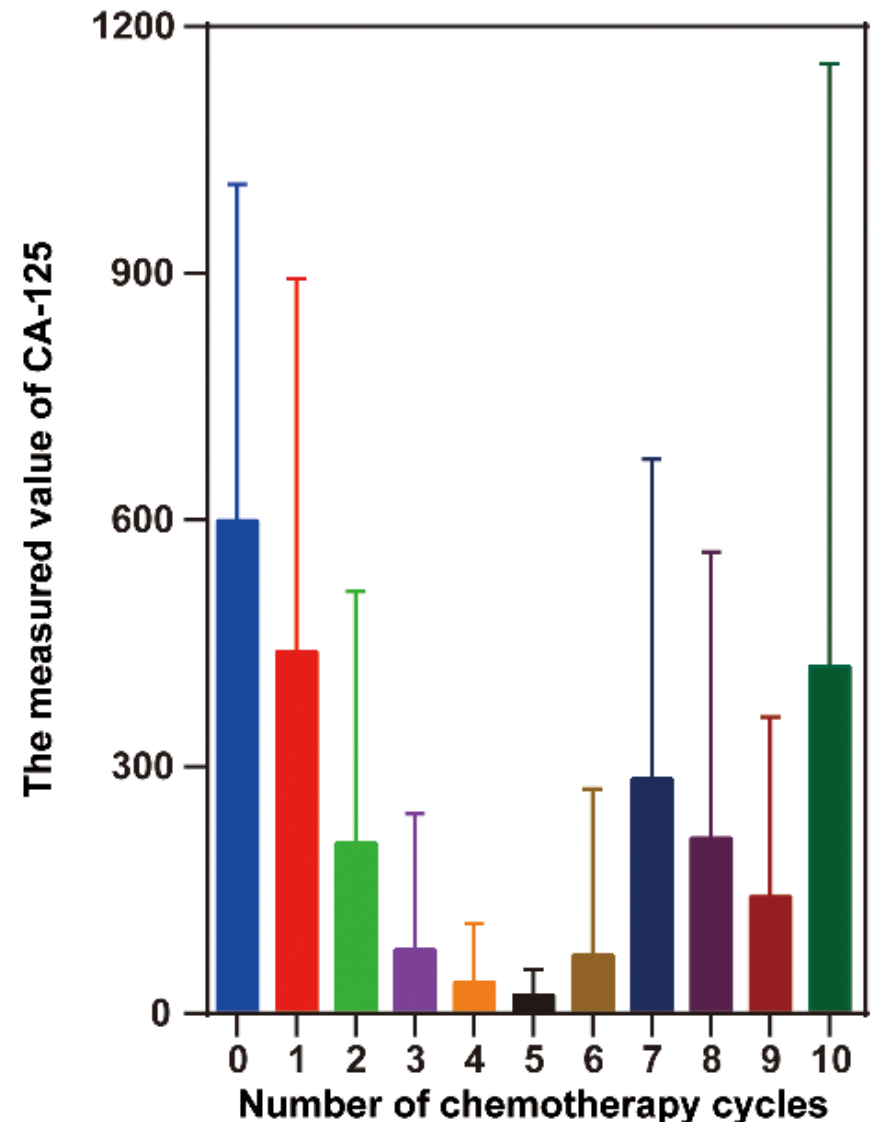

B

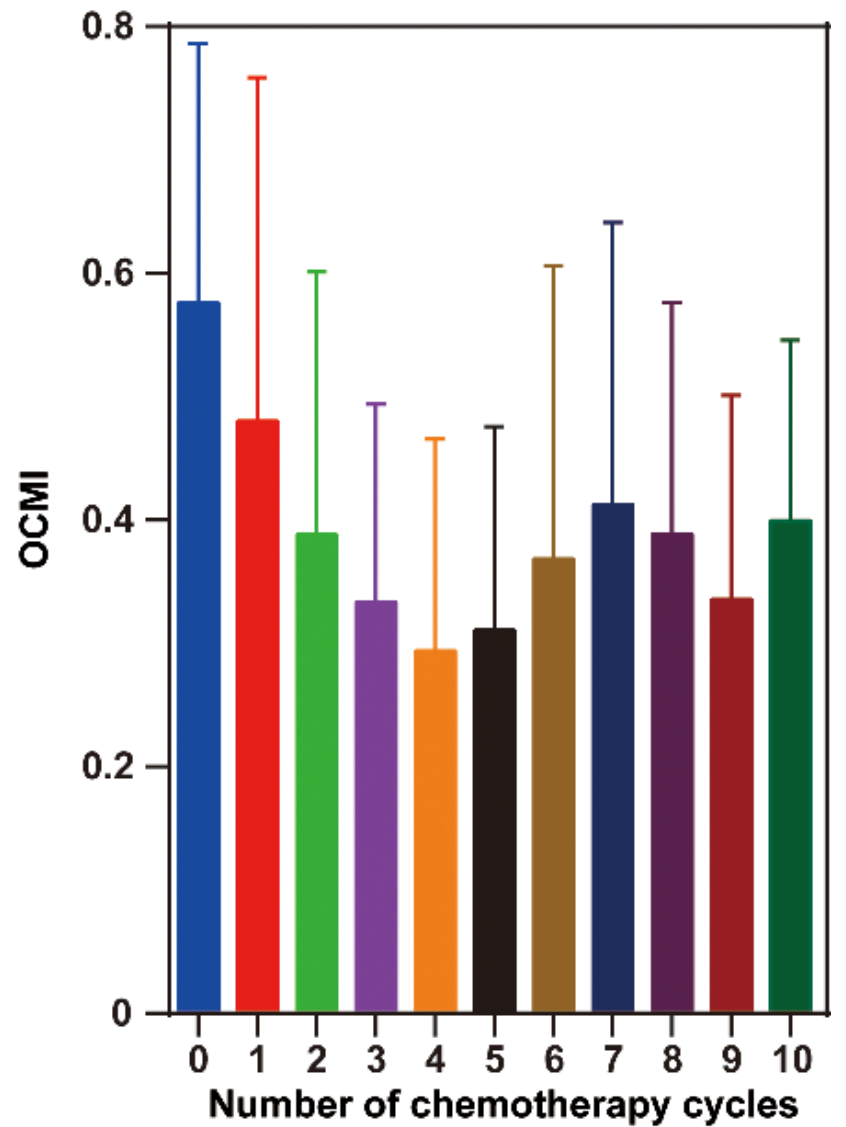

C

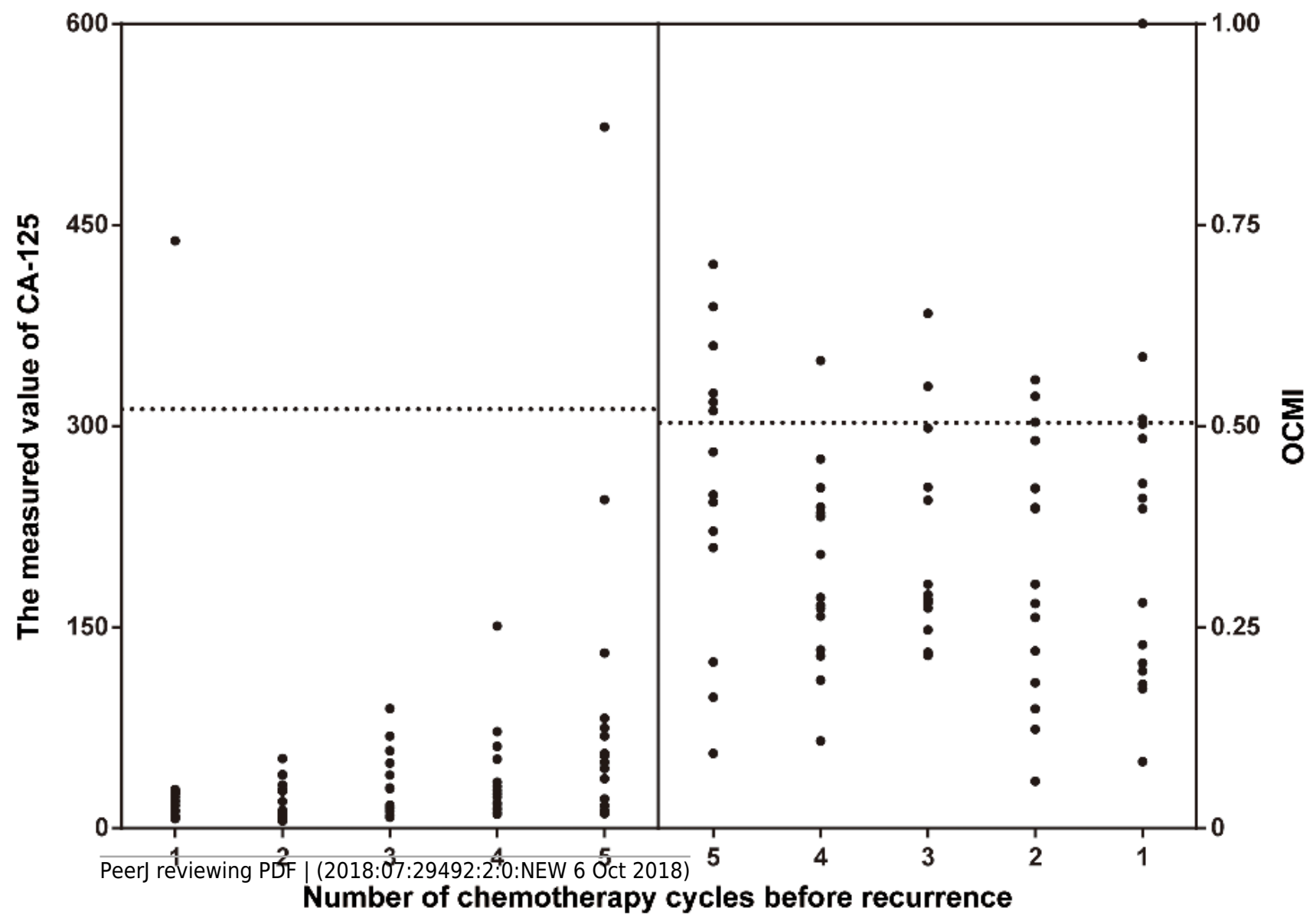

